\title{
МИНИМИЗАЦИЯ ДОМИНИРУЮЩЕЙ ПОГРЕШНОСТИ В ЗАДАЧАХ ИЗМЕРЕНИЯ ИНФОРМАЦИОННЫХ ПАРАМЕТРОВ «ЗАШУМЛЕННОЙ» ВЫБОРКИ СИГНАЛА
}

\author{
Скачков В.В., Чепкий В.В., Ефимчиков А.Н. \\ Военная академия, Одесса, Украина \\ Братченко Г.Д., Павлович В.И.
}

Одесская государственная академия технического регулирования и качества, Одесса, Украина

Background. The task of informative parameters of radio engineering signal measurement in the case of domination in target result error the component that generated by additive noises of an external environment of measurement is characteristic for many applications of radio engineering, monitoring of the environment, monitoring and technical diagnostics. The optimal solution of "weight's" minimization of the dominating error in mean squared sense as a result of measurement for the purpose of its approximation to the reference is considered.

Objective. The aim of the paper is the application of preprocessing with the adaptive filtering of additive noises in the task of minimization of the dominating measurement error of informative parameters of "noisy" sampling of a radio engineering signal.

Methods. Scientific novelty of work consists in modification of the form of the operator measurement's equation of information parameters considering specifics of procedure of bleaching. The problem of preprocessing of observed selection for automatic matching of measurement's procedure with input signal parameters and receiving the maximum number of information on an input of measuring instrument is solved. Efficiency of such preprocessing is estimated by information criterion which generalized measure is the modified entropy metrics according to Shannon.

Results. Problem situations in informative parameters measurement tasks of a radio engineering signal against additive noises of arbitrary intensity are considered. Operator and algorithmic forms of the modified measurement equation of signal's information parameters, and also option of their implementation in the form of the device of the preprocessing with the adaptive filtering performed according to the diagram of the linear weight summing are provided. Efficiency of the adaptive filtering "noisy" sampling of a measuring signal is estimated and validity of operation of preprocessing which allows minimizing information losses on measurement instrument input is confirmed.

Conclusions. Features of the measuring task solution are investigated and propositions on application of the preprocessing technology with the adaptive filtering additive noises of an external environment for minimization of "weight" of the dominating error in the result of measurement are formulated.

Keywords: informative parameters; result of measurement; error; operator equation; adaptive filtration; entropy metrics.

\section{Введение}

Задача измерения информационных параметров радиотехнического сигнала при доминировании в целевом результате погрешности, порождаемой аддитивными шумами внешней среды, характерна для многих приложений радиотехники, мониторинга среды, контроля и технической диагностики. Естественным подходом к решению такого класса измерительных задач является минимизация «веса» доминирующей погрешности в результате измерения с целью приближения его к своему эталону в среднеквадратическом смысле [1-3].

В общеизвестной постановке оптимальное решение задачи измерения параметров радиотехническо- го сигнала $S\left(t, \boldsymbol{\alpha}_{S}\right)$, представленных вектором $\boldsymbol{\alpha}_{S}$, которые содержат информацию об измеряемой физической величине $\lambda_{S}$, предполагает проведение анализа свойств измерительной процедуры и получаемого результата.

Соотношение, устанавливающее связь результата измерений $\tilde{\lambda}_{S}$ с входным воздействием (измерительным сигналом [2]) $S\left(t, \boldsymbol{\alpha}_{S}\right)$, известное в метрологии как операторная форма классического уравнения измерения [3-5], имеет следующий вид:

$$
\tilde{\lambda}_{S}=L\left\{S\left(t, \boldsymbol{\alpha}_{S}\right)\right\}=\lambda_{S}+\Delta \lambda(\varepsilon),
$$

где $L\{*\}$ - оператор нелинейного преобразования 
измерительного сигнала в результат измерения $\tilde{\lambda}_{S}$; $\lambda_{S}$ - действительное значение измеряемой физической величины; $\Delta \lambda(\varepsilon)$ - случайная погрешность результата измерения в условиях заданной точности измерительного средства $\varepsilon$.

Выражая базовые принципы процесса измерения, операторное уравнение (1), не учитывает стохастического состояния среды измерений, порождаемого независимыми внешними аддитивными шумами $N(t)$ произвольной интенсивности, не отражает реальную ситуацию измерительной процедуры и, следовательно, не может быть использовано для принятия оптимального решения. В связи с этим, возникает потребность в модификации классического уравнения измерения (1), учитывающей многообразие ситуационных состояний, которые характеризуются следующей проблематикой [6-9]:

- во-первых, особенностями взаимодействия средств измерения с дистанционно-удаленным объектом посредством радиотехнического сигнала $S\left(t, \boldsymbol{\alpha}_{S}\right)$, информационные параметры $\boldsymbol{\alpha}_{i}, i=\overline{1, \mu}$ которого трансформируются под влиянием характеристик объекта измерения и среды его пребывания;

- во-вторых, зависимостью целевого результата измерений $\tilde{\lambda}_{S N}$ от степени “зашумленности” выборки измеряемого случайного процесса $U(t)=S\left(t, \boldsymbol{\alpha}_{S}\right)+N(t)$ в виде аддитивной смеси полезного сигнала $S\left(t, \boldsymbol{\alpha}_{S}\right)$ (квазидетерминированная составляющая) и шума $N(t)$ (случайная составляющая). В присутствии аддитивных шумов указанная зависимость представляется операторным уравнением измерения следующего вида $[6,7]$ :

$$
\tilde{\lambda}_{S N}=L\{U(t)\}=L\left\{S\left(t, \boldsymbol{\alpha}_{S}\right)+N(t)\right\}+\Delta \lambda(\varepsilon) .
$$

При аппроксимации оператора преобразования $L\{*\}$ ограниченным рядом Тейлора, выражение (2) можно представить как

$$
\tilde{\lambda}_{S N}=\lambda_{S}+\Delta \lambda\{N(t), \varepsilon\},
$$

где $\Delta \lambda\{N(t), \varepsilon\}=\Delta \lambda\{N(t)\}+\Delta \lambda(\varepsilon)-$ суммарная погрешность целевого результата, которая состоит из случайной погрешности средства измерения $\Delta \lambda(\varepsilon)$ и независимой случайной погрешности измерительного сигнала $\Delta \lambda\{N(t)\}, \quad$ порождаемой внешними аддитивными шумами $N(t) ; \tilde{\lambda}_{S N} \in \boldsymbol{\alpha}_{S N}$.
Здесь $\boldsymbol{\alpha}_{S N}$ - вектор информационных параметров радиосигнала $S\left(t, \boldsymbol{\alpha}_{S N}\right)$, искаженных шумами;

- в-третьих, "реакцией” измерительных средств не только на измеряемую физическую величину $\lambda_{S}$, но и на воздействие многофакторных параметров возмущенной среды, которое, в общем случае, может привести к увеличению суммарной погрешности результата измерения $\Delta \lambda\{N(t), \varepsilon\}$;

- в-четвертых, устойчивой связью между уровнем “зашумленности” измерительного сигнала $S\left(t, \boldsymbol{\alpha}_{S N}\right)$ и среднеквадратической погрешностью результата измерения $E_{S}(t)$ (Рис. $1, \mathrm{a}$, б). Нестабильность характера “зашумленности” $N(t)$ и изменчивость его параметров относительно состояния среды измерения приводят к доминированию погрешности $\Delta \lambda\{N(t)\}>\Delta \lambda(\varepsilon)$, к росту суммарной погрешности целевого результата $\Delta \lambda\{N(t), \varepsilon\}$ и, как следствие, к низкой информационной достоверности данных, наблюдаемых на входе средства измерения [6, 9-11];

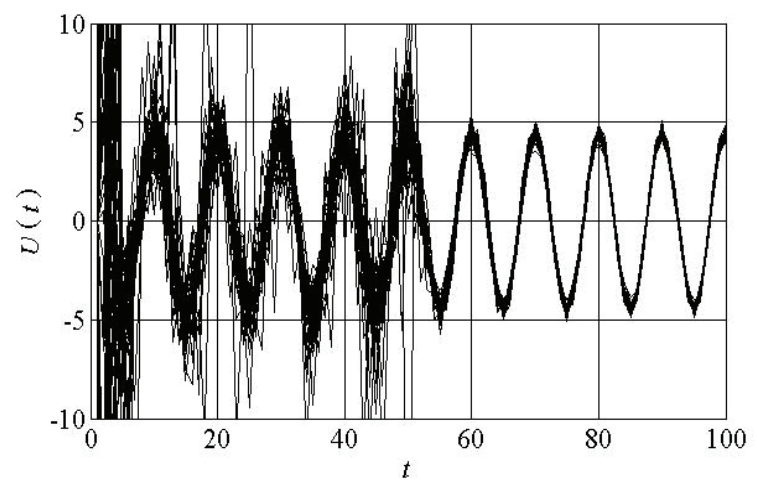

a)

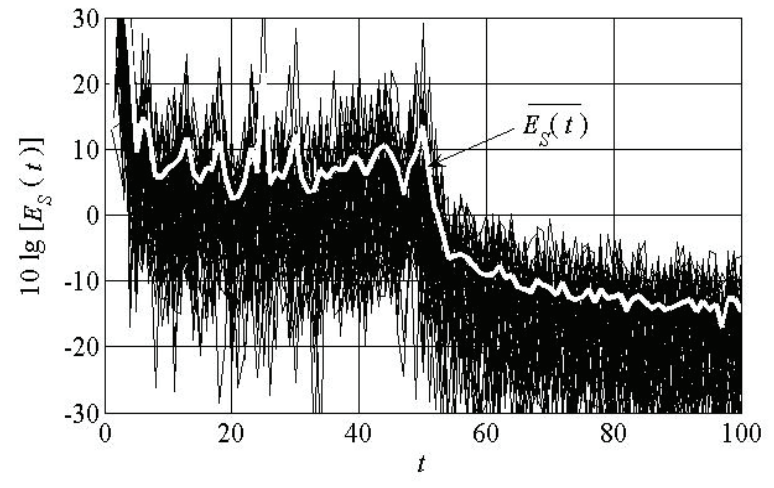

б)

Рис. 1. Иллюстрация влияния “зашумленности” измерительного сигнала (а) на среднеквадратическую погрешность $E_{S}(t)$ результата измерения (б) 
- в-пятых, существованием области неопределенности, как свойства общего по отношению к качественной оценке результата измерения $\lambda_{S N}$ и различным ситуациям, так и индивидуального по отношению к количественной оценке вектора информационных параметров $\boldsymbol{\alpha}_{S}$ измерительного сигнала $S\left(t, \boldsymbol{\alpha}_{S}\right)[6,7,10]$.

\section{Постановка задачи}

Целью работы является применение предварительного преобразования с адаптивной фильтрацией аддитивных шумов в задаче минимизации доминирующей погрешности измерения информационных параметров “зашумленной” выборки радиотехнического сигнала.

В контексте обозначенной целевой установки, как следует из $[3,6,7,11,12]$, необходимо модифицировать операторное уравнение измерения (2), представив его в аддитивной форме. Соответственно, целевой результат измерения, учитывающий технологию операций преобразования (фильтрации) $\lambda_{S N}^{F}$, можно записать в следующем виде [10, 12]:

$$
\tilde{\lambda}_{S N}^{F}=L\left\{F\left[S\left(t, \boldsymbol{\alpha}_{S}\right)+N(t)\right]\right\} \Rightarrow L\left\{S\left(t, \boldsymbol{\alpha}_{S N}\right)\right\}=\tilde{\lambda}_{S}
$$

где $F\left[S\left(t, \boldsymbol{\alpha}_{S}\right)+N(t)\right]$ - оператор предварительного линейного преобразования наблюдаемого процесса $U(t)$, которое направлено на подавление аддитивных шумов произвольной интенсивности [12-15].

Известные свойства линейного преобразования [13], позволяют переписать уравнение измерения (3), в такой расстановке:

$$
\begin{aligned}
& \tilde{\lambda}_{S N}^{F}=L\left\{F\left[S\left(t, \boldsymbol{\alpha}_{S}\right)\right]+F[N(t)]\right\} \Rightarrow \\
& \Rightarrow L\left\{S\left(t, \boldsymbol{\alpha}_{S N}\right)\right\}=\tilde{\lambda}_{S}
\end{aligned}
$$

Вид операторного уравнения измерений (4) определяется значимостью и местом расположения преобразования $F[U(t)]$. Если преобразование $F[U(t)]$ гипотетически реализует операцию усреднения для измерения вероятностных характеристик случайных процессов, то оно относится к ряду главных (основных) измерительных преобразований. В ином случае это преобразование является вспомогательным (предварительным) и фактически направлено на подавление (фильтрацию) аддитивных шумов с неизвестными статистическими свойствами $[1,3,6]$. В контексте реализации операции параметрической адаптации оператор преобра- зования $F[*]$ должен обладать такими свойствами:

- во-первых, свести к минимуму влияние аддитивных шумов $N(t)$ произвольной интенсивности на результат измерения $\tilde{\lambda}_{S N}^{F}$

$$
F[N(t)] \Rightarrow \min ;
$$

- во-вторых, минимизировать искажение вектора информационных параметров $\boldsymbol{\alpha}_{S N}$ наблюдаемых радиотехнических сигналов, то есть отображать в себя множество полезных сигналов

$$
F\left[S\left(t, \boldsymbol{\alpha}_{S N}\right)\right] \Rightarrow S\left(t, \boldsymbol{\alpha}_{S}\right) ;
$$

- в-третьих, соответствовать результату измерения физических параметров. Если норма линейного оператора $\left\|F\left[S\left(t, \boldsymbol{\alpha}_{S N}\right)\right]\right\|$ конечна, то результаты преобразования ограничены по величине некоторым вещественным положительным числом $\varepsilon$, а именно, заданной точностью измерения

$$
\left\|F\left[S\left(t, \boldsymbol{\alpha}_{S N}\right)\right]\right\|<\varepsilon .
$$

Исходя из реализации указанных свойств среднеквадратическая погрешность результата измерения $\tilde{\lambda}_{S N}^{F}$ будет равна

$$
E_{S N}^{F}=\sqrt{\left|\tilde{\lambda}_{S N}^{F}-\tilde{\lambda}_{S}\right|^{2}} \Rightarrow E_{S} .
$$

Очевидно, что при неограниченном времени измерения и бесконечном объеме выборки входных реализаций $U(t)$ операцией предварительного преобразования $F[U(t)]$ можно было бы пренебречь. В таком случае, подобная ситуация выходит за рамки физических возможностей решаемой измерительной задачи. Конструктивный подход к преодолению указанного ограничения предусматривает применение в измерительной процедуре преобразований с адаптивной фильтрацией $F[U(t)]$. Адаптация предполагает автоматическое согласование алгоритма измерения со свойствами входного воздействия с целью обеспечения максимума информации на входе измерительного средства. Для оценки информационных возможностей преобразования $F[U(t)]$ необходимо перейти от операторного уравнения измерения (4) к алгоритмическому.

\section{Основная часть}

Физически реализация предварительного преоб- 
разования $F[U(t)]$ требует переформатирования уравнения измерения (4), в частности, представления его в алгоритмической форме. Это позволяет описать операцию адаптивной фильтрации в виде свертки импульсной характеристики системы $h(t)$ и наблюдаемого процесса $U(t)$ :

$$
Y(t)=F[U(t)]=\int_{-\infty}^{\infty} h(t-\tau) U(\tau) \mathrm{d} \tau .
$$

С целью определения базисной системы преобразования представим импульсную характеристику $h(t)$ конечным временным рядом

$$
h_{\Delta}(t)=\sum_{i=1}^{m} w(i \Delta t) \delta(t-i \Delta t)
$$

где $\Delta t-$ интервал дискретизации; $\delta(t)$ - дельтафункция или функция Дирака.

В этом случае интеграл сверки (5) сводится к следующему виду:

$$
Y_{\Delta}(t)=\sum_{i=1}^{m} w_{i} \int_{-\infty}^{\infty} \delta[(t-i \Delta t)-\tau] U(\tau) \mathrm{d} \tau,
$$

где $w_{i}=w(i \Delta t)-$ весовые коэффициенты.

C учетом фильтрующего свойства $\delta$-функции выражение (6) можно аппроксимировать весовой суммой:

$$
Y_{\Delta}(t)=U_{\Sigma}=\sum_{i=1}^{m} w_{i} U(t-i \Delta t)=\mathbf{W}^{\mathrm{T}} \mathbf{U},
$$

где “Т” - символ операции транспонирования; $\mathbf{U}=\left[\begin{array}{llll}U(t) & U(t-\Delta t) & \ldots & U(t-m \Delta t)\end{array}\right]^{\mathrm{T}}-$ векторстолбец процессов на входе системы преобразования; $\mathbf{W}=\left[\begin{array}{llll}w_{1} & w_{2} & \ldots & w_{m}\end{array}\right]^{\mathrm{T}}-$ параметрический вектор весовых коэффициентов $w_{i}, i=\overline{1, m}$, который удовлетворяет требуемому критерию оптимальности $[1,3-7,16,17]$

$$
\left|Y(t)-Y_{\Delta}(t)\right|^{2} \Rightarrow \min .
$$

Из аналитического выражения (7) следует, что функционально операцию преобразования $F[U(t)]$ можно выполнить по схеме линейного весового суммирования (Рис. 2), физически реализуя ее как адаптивный трансверсальный фильтр.

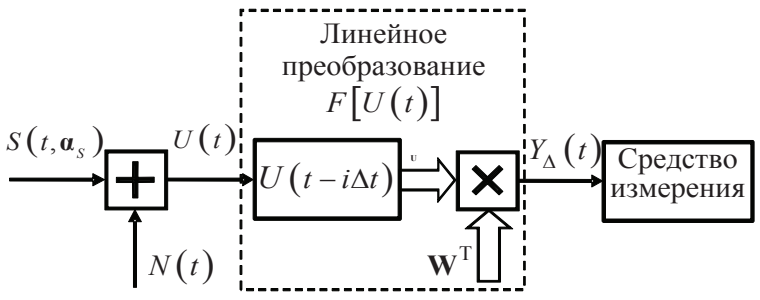

Рис. 2. Схема линейного весового суммирования
Пример решения измерительной задачи с применением адаптивного трансверсального фильтра для минимизации “веса” доминирующей составляющей $\Delta \lambda\{N(t)\}$ в суммарной погрешности результата измерения $\tilde{\lambda}_{S N}$ приведен на Рис. 3. Представленные на Рис. 3 результаты статистического моделирования показывают, что предварительное преобразование с адаптивной фильтрацией “зашумленной” выборки измеряемого случайного процесса, обладая свойством “отбеливания", позволяет “очистить" измерительный сигнал от воздействия шумовой составляющей, минимизировать влияние аддитивных шумов $N(t)$ на результат измерения, приближая целевой результат $\tilde{\lambda}_{S N}$ к его эталону $\tilde{\lambda}_{S}$, то есть: $\tilde{\lambda}_{S N} \rightarrow \tilde{\lambda}_{S}$.

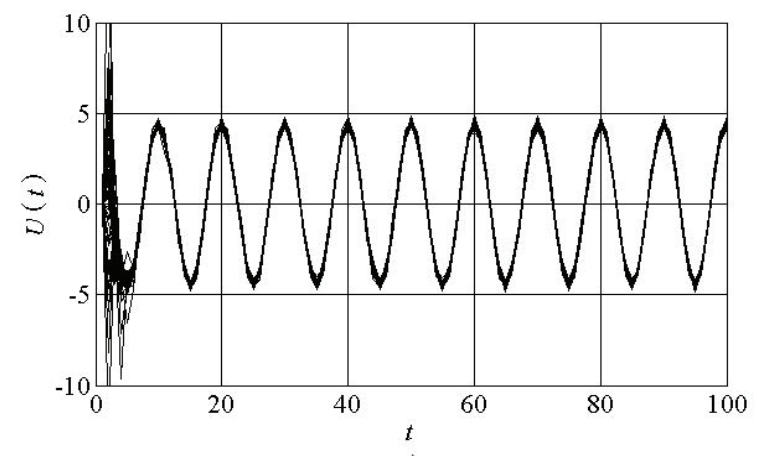

a)

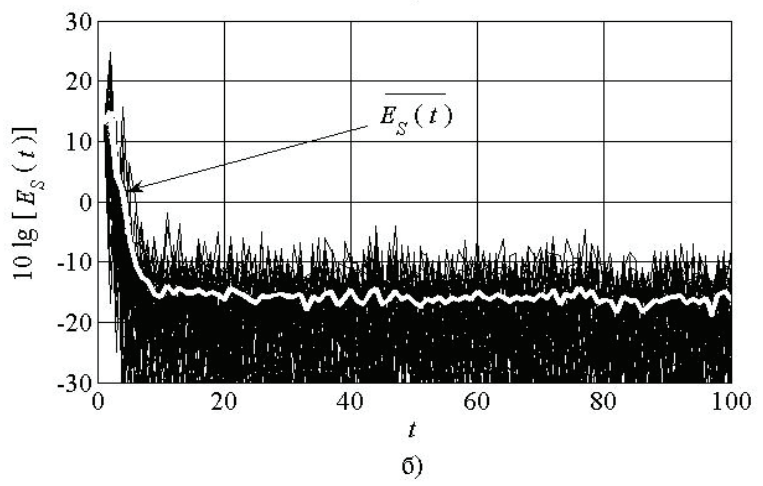

Рис. 3. Иллюстрация влияния процедуры “отбеливания” на измерительный сигнал (а) и среднеквадратическую погрешность $E_{S}(t)$ результата измерения (б)

В современных средствах измерения на вход фильтра предварительного преобразования $F[U(t)]$ поступает сигнал измерительной информации. Поэтому эффективность адаптивной фильтрации “зашумленной” выборки входного сигнала $U(t)$, естественно оценить информационным кри- 
терием. Обобщенной мерой этого критерия, как известно [6, 10, 11, 18], служит энтропийная метрика по Шеннону, модифицированная к условиям решаемой измерительной задачи

$$
I\left(S_{\Sigma}, U_{\Sigma}\right)=H\left(U_{\Sigma}\right)-H\left(U_{\Sigma} / S_{\Sigma}\right)
$$

где $S_{\Sigma}=F\left[S\left(t, \boldsymbol{\alpha}_{S}\right)\right]=\mathbf{W}^{\mathrm{T}} \mathbf{S}-$ проекция преобразования $F[U(t)]$ на плоскость полезных сигналов $\mathbf{S}$; $H\left(U_{\Sigma}\right)$ - потенциальная энтропия процесса на входе средства измерения; $H\left(U_{\Sigma} / S_{S}\right)$ - условная (апостериорная) энтропия процесса на входе средства измерения при наличии полезного сигнала $S\left(t, \boldsymbol{\alpha}_{S}\right)$.

Адаптивное преобразование $F[U(t)]$, при котором достигается экстремум требуемого критерия оптимальности $J\{F[U(t)]\}$, предполагает фильтрацию “зашумленной” выборки наблюдений $U(t)$. Решение задачи оптимального преобразования $F[U(t)]$ и вычисления среднего количества информации $I\left(S_{\Sigma}, U_{\Sigma}\right)$ непосредственно и достаточно подробно изложено в $[1,10,18]$.

Используя результаты этих исследований, определим теоретический предел оценки информационных возможностей преобразования $F[U(t)]$, когда шум с фиксированным значением средней мощности обладает наибольшей энтропией. Последнее адекватно максимальным потерям измерительной информации на входе средства измерения при наличии $m$-мерного адаптивного фильтра

$$
I\left(S_{\Sigma}, U_{\Sigma}\right)=0,5 \lg \left[1+\frac{\mathbf{W}^{\mathrm{T}} \mathbf{A}_{S} \mathbf{W}}{\mathbf{W}^{\mathrm{T}}\left(\mathbf{A}_{J}+\mathbf{A}_{n}\right) \mathbf{W}}\right]
$$

где $\frac{\mathbf{W}^{\mathrm{T}} \mathbf{A}_{S} \mathbf{W}}{\mathbf{W}^{\mathrm{T}}\left(\mathbf{A}_{J}+\mathbf{A}_{n}\right) \mathbf{W}}-$ обобщенный параметр превышения уровня сигнала над внешней помехой и внутренним шумом на выходе $N$-мерной адаптивной системы; $\mathbf{A}_{S}, \mathbf{A}_{j}, \mathbf{A}_{n}$ - ответственно, матрицы полезного сигнала, внешней помехи, внутреннего шума, суммарно представляющие корреляционную матрицу А.

Приведенное к уровню внутренних шумов выражение (8) принимает вид:

$$
I\left(S_{\Sigma}, U_{\Sigma}\right)=0,5 \lg \left[1+\frac{q_{S}(\mathbf{W})}{1+q_{J}(\mathbf{W})}\right]
$$

где $q_{S}(\mathbf{W})$ и $q_{J}(\mathbf{W})$ - параметры, определяющие превышение сигнала и внешних аддитивных шумов (помех) над внутренними шумами на входе средства измерения, соответственно.

При отсутствии режима адаптации параметрический вектор имеет единичное значение $\mathbf{W}=\mathbf{I}(m, 1)$, что соответствует количеству информации

$$
I\left(S_{\Sigma}, U_{\Sigma}\right)=0,5 \lg \left\{1+m \frac{q_{S}}{1+q_{J}}\right\} .
$$

Теоретический предел оценки информационных возможностей предварительного преобразования $F[U(t)]$ естественно рассматривать для винеровского параметрического вектора $\mathbf{W}=\mathbf{A}^{-1} \mathbf{r}[11,12$, 19]. Здесь $\mathbf{r}=E\left(U_{0} \mathbf{U}\right)$ - вектор взаимной корреляции между скалярным опорным (эталонным) процессом $U_{0}$ и вектором “зашумленной” выборки измеряемого случайного процесса $\mathbf{U}$.

Именно к оптимальному решению ВинераХопфа с точностью до постоянного множителя сходятся решения оптимизационных задач при синтезе адаптивных фильтров по критериям минимума среднеквадратической ошибки, максимума отношения сигнал/шум на выходе адаптивной системы и максимума функции правдоподобия [1, 3, 15, 19]. Особенности формирования опорного процесса $U_{0}$ в адаптивных системах различного назначения подробно рассмотрены в публикациях [14-16].

В контексте винеровского решения выражение (8), определяющее количество информации на выходе адаптивной системы, приобретает вид:

$$
I\left(S_{\Sigma}, U_{\Sigma}\right)=0,5 \lg \left[1+\frac{\mathbf{r}^{\mathrm{T}} \mathbf{A}^{-1} \mathbf{A}_{S} \mathbf{A}^{-1} \mathbf{r}}{\mathbf{r}^{\mathrm{T}} \mathbf{A}^{-1}\left(\mathbf{A}_{J}+\mathbf{A}_{n}\right) \mathbf{A}^{-1} \mathbf{r}}\right],
$$

Построенные по выражениям (10) и (11) семейства кривых (Рис. 4) иллюстрируют количество информации на входе средства измерения в зависимости от отношения помеха/шум $q_{J}$, при $q_{S}=10 \mathrm{~dB}$ и размерности адаптивного фильтра $m=3 ; 4 ; 10$. Обозначенные штриховыми линиями $I\left(S_{\Sigma}, U_{\Sigma}\right)=\varphi\left(q_{J}\right)$ кривые отображают динамику информационных потерь в условиях отсутствия адаптивной фильтрации, а сплошными линиями $I\left(S_{\Sigma}, U_{\Sigma}\right)=\varphi\left(q_{J}\right)-$ информационные потери при наличии таковой.

Анализ графиков семейства кривых, приведенных на Рис. 4, показывает, что:

- предварительное преобразование с адаптив- 
ной фильтрацией позволяет существенно уменьшить информационные потери, вызванные воздействием внешних факторов шумовой природы;

- уровень информационных потерь можно свести к минимуму путем увеличения размерности адаптивного фильтра;

- потенциальные возможности адаптивной фильтрации внешних шумов определяются присутствием в измерительной системе исключительно аддитивных внутренних шумов.

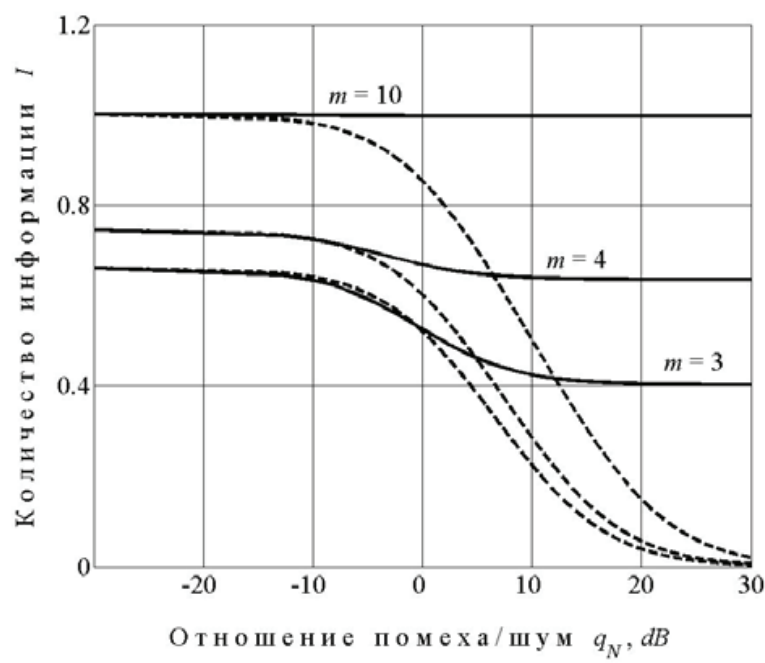

Рис. 4. Потери измерительной информации на входе средства измерения

\section{Заключение}

В развитие концепции решения проблемы измерительной задачи с доминирующей погрешностью в целевом результате измерения, порожденной аддитивными шумами внешней среды, предложено выполнить предварительную фильтрацию “зашумленной” выборки входного сигнала. Следуя этой логике, модифицировано и представлено в операторной и алгоритмической форме уравнение измерения информационных параметров радиотехнического сигнала, а также предложен предпочтительный вариант его реализации в виде предварительного адаптивного фильтра, выполненного по схеме линейного весового суммирования.

Оценена эффективность адаптивной фильтрации «зашумленной» выборки измерительного сигнала, которая показала, что операция предварительного преобразования позволяет свести к минимуму уровень информационных потерь на входе средства измерения, вызванных воздействием внешних факторов шумовой природы.

Обобщая сказанное, считаем необходимым в приложении к решению целевой измерительной задачи сделать ряд уточняющих комментариев следующего содержания.

1. Специфика целевой измерительной задачи состоит в понимании результата измерений как последовательности преобразований, увязывающих его с информационными параметрами $\boldsymbol{\alpha}_{S}$ радиотехнического сигнала $S\left(t, \boldsymbol{\alpha}_{S}\right)$. При этом процессу непосредственных измерений должна предшествовать процедура преобразования с адаптивной фильтрацией $F[U(t)]$ “зашумленной” выборки входного сигнала $U(t)$, обеспечивающая согласование измерительной процедуры с особенностями ее выполнения.

2. Представленная весовой суммой аппроксимация оператора преобразования $F[U(t)]$, независимо от физического приложения, должна обладать свойствами, которые способствуют достижению следующих результатов:

- трансформирования полезного радиотехнического сигнала на вход средства измерения без каких-либо искажений $\mathbf{W}^{\mathrm{T}} \mathbf{S}=S_{\Sigma}$;

- минимизации влияния аддитивных шумов $N(t)$ с неизвестными статистическими свойствами на входе средства измерений;

- принадлежности целевой измерительной задачи к классу корректных задач, то есть физически реализуемой в пределах заданной точности аппроксимации операторного преобразования при условии $\|\mathbf{W}\|^{2}<\varepsilon$, где $\varepsilon<\infty$.

3. Информационные потери можно свести к минимуму без предварительного преобразования $F[U(t)]$ “зашумленной” выборки входной реализации $U(t)$ только при неограниченном временном ресурсе на решение измерительной задачи.

4. Применение адаптивной фильтрации параметров “зашумленной” выборки радиотехнического сигнала является естественным направлением совершенствования современных технологий в теории и практике построения информационноизмерительных систем различного назначения.

\section{Литература}

1. Репин В. Г. Статистический синтез при априорной неопределённости и адаптация информационных систем / В. Г. Репин, Г. П. Тартаковский.- М.: Сов. радио, 1977.- 404 c. 
2. Сигнали радіотехнічні вимірювальні. Терміни та визначення. ДСТУ 3592-97. [Чинний від 1998-07-01].Київ: Держстандарт України, 1997.- 29 с.

3. Цветков Э. И. Основы математической метрологии / Э. И. Цветков. - СПб.: Политехника, 2005. - 510 с.

4. Царев В. А. Неконтактные методы измерения в океанологии: Учебное пособие / В.А. Царев, В.П. Коровин. - СПб.: изд. РГГМУ, 2005. - 184 с.

5. Жиганов И. Ю. Метрологические основы дистанционных методов измерения геометрических параметров объектов / И. Ю. Жиганов // Физика волновых процессов и радиотехнические системы. - 2009. - Т. 12, № 4. C. $83-87$.

6. Скачков В. В. Проблема повышения качества измерения информативных параметров радиосигналов в условиях аддитивных шумов / В. В. Скачков, В. И. Павлович // Метрологія технічне регулювання та забезпечення якості: матеріали П'ятої Міжнародної науковопрактичної конференції, 08-09 жовтня 2015 р. - Одеса: ОДАТРЯ, 2015. - С. 96-99.

7. Павлович В. И. Формализация задачи измерения информационных параметров радиосигналов при условии адаптивной фильтрации аддитивных шумов / В. И. Павлович // Цифровые технологии: Сб. науч. тр. Одесса: ОНАС, 2015. - Вып. 18. - С. 53-65.

8. Коробейников С. А. Параметрическая оптимизация решающего правила при адаптивных измерениях / С. А. Коробейников // Вестник Северо-Западного отделения Метрологической академии. - 2002. - Вып. 9. C. $67-72$.

9. Вельтищев Н.Ф. Дистанционные методы измерения в гидрометеорологии: Учеб. пособие / Н.Ф. Вельтищев, Б.А. Семенченко. - М.: изд. Московский университет, 2005. - 129 с

10. Скачков В.В. Энтропийный подход к исследованию информационных возможностей адаптивной радиотехнической системы при внутрисистемной неопределенности /В. В. Скачков, В. В. Чепкий, Г. Д. Братченко, А. Н. Ефимчиков // Известия вузов. Радиоэлектроника. 2015. - T. 58, № 6. - C. 3-12.
11. Скачков В.В. Энтропийное оценивание влияния внутрисистемных возмущений на информационные возможности адаптивной радиотехнической системы / В. В. Скачков, В. В. Чепкий, А. Н. Ефимчиков, Г. Д. Братченко, В.И.Павлович // Сборник научных трудов в 4-х томах. Том 4. Конференция «Интегрированные информационные радиоэлектронные системы и технологии. - Харьков: АНПРЭ, ХНУРЭ, 2014. - С. 55-59.

12. Скачков В.В. Оценка влияния внутрисистемных возмущений на точность измерения пространственных частот методом максимальной энтропии / В. В. Скачков, В. В. Чепкий, А. Н. Ефимчиков, В. И. Павлович // Системы обработки информации: сборник научных прудов. Харьков: ХУПС, 2015. - Вып. 6 (131). - С. 131-134.

13. Френкс Л. Теория сигналов / Л. Френкс; пер. с англ. под ред. Д. Е. Вакмана. - М: Сов. радио, 1974. $344 \mathrm{c}$.

14. Адаптивные фильтры: пер. с англ. / П.М. Грант, К.Ф.Н. Коуэн, Б. Фридлендер и др.; под ред. К.Ф.Н. Коуэна и П.М. Гранта. - М.: Мир, 1988. - 392 с.

15. Цветков Э. И. Измерения с адаптивной фильтрацией аддитивной помехи / Э. И. Цветков // Вестник Вестник Северо-Западного отделения Метрологической академии. - 2004. - Вып. 12. - С. 4-16.

16. Черемисин О.П. Эффективность адаптивного алгоритма с регуляризацией выборочной корреляционной матрицы / О. П. Черемисин // Радиотехника и электроника. - 1982. - Том 27, №10. - С. 1933-1942.

17. Гиг Дж. Ван. Прикладная общая теория систем / Гиг Дж. Ван: пер. с англ. - М.: Мир, 1981. - 336 с.

18. Шеннон К. Работы по теории информации и кибернетике / К. Шеннон; пер с англ. - М.: Изд-во иностр. лит., 1963. -830 с.

19. Ван Трис Г. Теория обнаружения, оценок и модуляции: в 4 т. / Г. Ван Трис; пер с англ. под ред. В.И. Тихонова. - М.: Сов. радио, 1972. - Т. 1. - 744 с.

Поступила в редакцию 16.09.2016

Скачков В.В., Чепкій В.В., Сфимчиков О.М., Братченко Г.Д., Павлович В.І. Мінімізація домінуючої похибки в задачах вимірювання інформаційних параметрів «зашумленої» вибірки сигналу

Проблематика. Задача вимірювання інформаційних параметрів радіотехнічного сигналу за умови домінування у похибці цільового результату складової, що породжена адитивними шумами зовнішнього середовища вимірювання, характерна для багатьох додатків радіотехніки, моніторингу середовища, контролю й технічної діагностики. Розглядається оптимальне в середньоквадратичному розумінні рішення щодо мінімізації «ваги» домінуючої похибки в результаті вимірювання з метою наближення його до свого еталона.

Мета. Застосування попереднього перетворення з адаптивною фільтрацією адитивних шумів у задачі мінімізації домінуючої похибки вимірювання інформаційних параметрів «зашумленої» вибірки радіотехнічного сигналу.

Методи. Наукова новизна роботи полягає в модифікації форми операторного рівняння вимірювання інформаційних параметрів, яка враховує специфіку процедури відбілювання. Вирішується задача попереднього перетворення вибірки, що спостерігається, для автоматичного узгодження процедури вимірювання з параметрами вхідного сигналу та одержання 
максимальної кількості інформації на вході вимірювального засобу. Ефективність такого перетворення оцінюється інформаційним критерієм, узагальненою мірою якого є видозмінена энтропійна метрика за Шенноном.

Результати. Розглянуті проблемні ситуації в задачах вимірювання інформаційних параметрів радіотехнічного сигналу на фоні адитивних шумів довільної інтенсивності. Наведені операторна та алгоритмічна форми модифікованого рівняння вимірювання інформаційних параметрів сигналу, а також варіант їх реалізації у вигляді пристрою попереднього перетворення з адаптивною фільтрацією, виконаного за схемою лінійного вагового підсумовування. Оцінена ефективність адаптивної фільтрації «зашумленої» вибірки вимірювального сигналу та підтверджена обгрунтованість операції попереднього перетворення, яка дозволяє мінімізувати інформаційні втрати на вході засобу вимірювання.

Висновки. Досліджені особливості розв’язання вимірювальної задачі та сформульовані пропозиції щодо застосування технології попереднього перетворення з адаптивною фільтрацією адитивних шумів зовнішнього середовища для мінімізації «ваги» домінуючої погрішності в результаті виміру.

Ключові слова: інформаційні параметри; результат виміру; погрішність; операторне рівняння; адаптивна фільтрація; ентропійна метрика.

Скачков В.В., Чепкий В.В., Ефимчиков А.Н., Братченко Г.Д., Павлович В.И.

Минимизация доминирующей погрешности в задачах измерения информационных параметров «зашумленной» выборки сигнала

Проблематика. Задача измерения информационных параметров радиотехнического сигнала при доминировании в погрешности целевого результата составляющей, порождаемой аддитивными шумами внешней среды измерения, характерна для многих приложений радиотехники, мониторинга среды, контроля и технической диагностики. Рассматривается оптимальное в среднеквадратическом смысле решение минимизации «веса» доминирующей погрешности в результате измерения с целью приближения его к своему эталону.

Цель. Применение предварительного преобразования с адаптивной фильтрацией аддитивных шумов в задаче минимизации доминирующей погрешности измерения информационных параметров «зашумленной» выборки радиотехнического сигнала.

Методы. Научная новизна работы заключается в модификации формы операторного уравнения измерения информационных параметров, учитывающей специфику процедуры отбеливания. Решается задача предварительного преобразования наблюдаемой выборки для автоматического согласования процедуры измерения с параметрами входного сигнала и получения максимального количества информации на входе измерительного средства. Эффективность такого преобразования оценивается информационным критерием, обобщенной мерой которого является видоизмененная энтропийная метрика по Шеннону.

Результаты. Рассмотрены проблемные ситуации в задачах измерения информативных параметров радиотехнического сигнала на фоне аддитивных шумов произвольной интенсивности. Приведены операторная и алгоритмическая формы модифицированного уравнения измерения информационных параметров сигнала, а также вариант их реализации в виде устройства предварительного преобразования с адаптивной фильтрацией, выполненного по схеме линейного весового суммирования. Оценена эффективность адаптивной фильтрации «зашумленной» выборки измерительного сигнала и подтверждена обоснованность операции предварительного преобразования, которая позволяет минимизировать информационные потери на входе средства измерения.

Выводы. Исследованы особенности решения измерительной задачи и сформулированы предложения по применению технологии предварительного преобразования с адаптивной фильтрацией аддитивных шумов внешней среды для минимизации «веса» доминирующей погрешности в результате измерения.

Ключевые слова: информационные параметры; результат измерения; погрешность; операторное уравнение; адаптивная фильтрация; энтропийная метрика. 